\title{
SCIENTIFIC CHALLENGES OF CONVECTIVE-SCALE NUMERICAL WEATHER PREDICTION
}

\author{
Jun-Ichi Yano, Michat Z. Ziemiański, Mike Cullen, Piet Termonia, Jeanette Onvlee, Lisa Bengtsson, \\ Alberto Carrassi, Richard Davy, Anna Deluca, Suzanne l. Gray, Victor Homar, Martin Köhler, \\ Simon Krichak, Silas Michaelides, Vaughan T. J. Phillips, Pedro M. M. Soares, \\ AND ANDRZEJ A. WYSZOGRODZKI
}

Numerical weather prediction models are increasing in resolution and becoming capable of explicitly representing individual convective storms, but we do not yet know if it is the improved resolution that is leading to better forecasts.

$\mathrm{T}$ he improvements in numerical weather prediction (NWP) over the last half century may overall be considered as an outcome of a straightforward extrapolation of technologies: increase of model resolution; relaxations of the dynamical approximations, from the quasigeostrophic to the primitive equation system, and with the removal of the hydrostatic balance approximation; introduction of more complex physics as well as parameterizations; ${ }^{1}$ and a more careful procedure for preparation of the forecast initial conditions. These model upgrades have been rather dramatic, thanks to an exponential growth in computer capabilities. In turn, these upgrades have contributed to the steady improvements of NWP forecast performance to date (cf. Bauer et al. 2015).

The effort to straightforwardly extrapolate technological capability has reached such a level that opera-

\footnotetext{
${ }^{1}$ Note that, unlike the common custom in atmospheric modeling, the present essay strictly distinguishes between physics and parameterizations: physics always refers to explicit physical processes, whereas parameterization always refers to subgrid-scale processes.
}

tional regional forecast models are now running with horizontal mesh sizes of $1-5 \mathrm{~km}$ worldwide. For example, in Europe, the French AROME (Applications de la Recherche à l'Opérationnel à Méso-Echelle) forecasts over France are run operationally with a grid spacing of $1.3 \mathrm{~km}$, the Met Office in the United Kingdom uses a grid spacing of $1.5 \mathrm{~km}$, and MeteoSwiss runs the Consortium for Small-Scale Modeling (COSMO) model with a grid spacing of $1.1 \mathrm{~km}$.

NWP capacity has reached a critical threshold: NWP models now begin to resolve individual convective elements within multicell, mesoscale, and synoptic-scale storms (i.e., they are "convection permitting" models). This tendency to higher resolution will continue: it is planned that the COSMO model will be run with a horizontal grid spacing of $500 \mathrm{~m}$ by 2020, and thus convection will be more resolved. A goal of convective-scale NWP is to accurately forecast high-impact storms, including their locations and intensities, which has the potential to bring a wide range of benefits to society. Forecast guidance from convective-scale NWP is already operationally available today. At the same time, this threshold also 
marks an end of straightforward extrapolation of technologies for NWP, even in the crudest sense: the convective-scale regime is very different from the well-studied synoptic weather regime, calling for a qualitatively different approach. The transition to forecasting at the convective scale is hardly a matter of straightforward extrapolation. There are several important gaps in our understanding: our basic and overall theoretical understanding of this regime is much weaker than for the synoptic-scale regime. The convective-scale regime is far more complex, even more so than as suggested by existing theoretical studies on convective dynamics (e.g., Moncrieff and Green 1972; Thorpe et al. 1982; Rotunno et al. 1988; Yano and Plant 2012).

Though specific issues for convective-scale NWP may be found discussed in the literature, the bigpicture view is missing: we can properly tackle the convective-scale NWP problems only by taking into account the full breadth of all the issues. Some of these challenges are particularly problematic: the convection-permitting regime is sometimes called the "gray zone," referring to a transition from a regime in which convection is fully parameterized to a regime in which convection is fully resolved, especially in the convection community. However, we should not reduce the problems of this regime just to that of convection parameterization. The extent of the challenge at the convective scale becomes apparent only when seeing all of the challenges together.

AFFILIATIONS: YANO-CNRM, CNRS and Météo-France, Toulouse, France; ZIEMIAŃSKI AND WYSZOGRODZKI-Institute of Meteorology and Water Management, National Research Institute, Warsaw, Poland; Cullen-Met Office, Exeter, United Kingdom; TERMONIA-Royal Meteorological Institute of Belgium, Brussels, Belgium; ONVLeE-KNMI, De Bilt, Netherlands; BeNGTSSONNOAA/CIRES, Boulder, Colorado; CARRASSI AND DAVY-NERSC, Bergen, Norway; Deluca-Institut Catal de Cincies del Clima, Barcelona, Spain; GrAY_University of Reading, Reading, United Kingdom; HomaR - Universidad de les Islas Baleares, Palma, Spain; KöHLER-DWD, Offenbach, Germany; KRICHAK-Tel Aviv University, Tel Aviv, Israel; MICHAELIDES-Cyprus Institute, Nicosia, and Cyprus University of Technology, Limassol, Cyprus; PHILLIPSUniversity of Lund, Lund, Sweden; SOARES-Instituto Dom Luiz, Faculdade de Ciências, Universidade de Lisboa, Lisbon, Portugal CORRESPONDING AUTHOR: Jun-Ichi Yano, jun-ichi.yano@meteo.fr

The abstract for this article can be found in this issue, following the table of contents.

DOI:10.1175/BAMS-D-17-0125.1

In final form 19 October 2017

(C)2018 American Meteorological Society

For information regarding reuse of this content and general copyright

information, consult the AMS Copyright Policy.
The practical issues faced by European weather services may be understood by the fact that, for example, a typical public user requirement in Switzerland would be a prediction of precipitation in a specific valley. A more specific example is a thunderstorm event at the Belgian music festival Pukkelpop in August 2011 (de Meutter et al. 2015). During the music festival, at which about 60,000 people were present, a short-lived downburst occurred. Five people were killed and at least 140 were injured. An operational failure to predict this downburst event was something to be criticized from a public perspective, although the downburst had a width of only $100 \mathrm{~m}$ and so was far too small to be resolved by current operational NWP models. ${ }^{2}$ Weather services naturally need to follow those public expectations. In responding to such expectations from the public, we also need to shift the focus to the finer scales and more fully exploit the information from convective-scale NWPs.

The present essay has emerged from a sense of an urgent need for action within European NWP consortia-Aire Limitée Adaptation Dynamique Développement International (ALADIN), COSMO, and High Resolution Limited Area Model (HIRLAM) - in responding to these challenges. This essay complements previous BAMS articles, including Mass et al. (2002), Fritsch and Carbone (2004), Mass (2006), Stensrud et al. (2009), and Sun et al. (2014). As discussed therein, we clearly acknowledge that currently there are extensive research efforts at the operational level to improve convective-scale NWP by exploiting various existing observations as well as modeling techniques. The main emphasis put forward in the present essay is an urgent need to properly address more fundamental theoretical issues. With our lack of basic understanding of this regime, current efforts will sooner or later otherwise become deadlocked. A good awareness of these more fundamental issues and of the limits of the current operational efforts is crucial just for good continuation of the current progress, even though those fundamental problems may not be immediately solvable.

To keep a reasonable focus, so that we can discuss the issues in depth, this essay addresses only the most basic theoretical issues. We recognize that other issues could be equally important, such as observation-related issues, but here we limit ourselves to only discussing these in the theoretical context. We

\footnotetext{
${ }^{2}$ See further discussion on the parameterization problems in the "Parameterization" section.
} 
clearly acknowledge the current operational efforts are of crucial importance, but for the sake of keeping focus, they are not covered herein.

In the next section, these fundamental issues are examined one by one. Discussions begin with the most basic issues of partial differential equations (PDEs), then turn to the issues of fluid mechanics, and then gradually move to more operational issues. Though the argument as a whole evolves over the section, since the issues to be discussed are so extensive each subsection on an issue is written in an almost stand-alone manner for ease of reading. In this manner, this essay provides a full breadth of the most fundamental problems of the convectivescale NWP.

\section{SCIENTIFIC CHALLENGES. Partial differential} equation problem. The synoptic weather system of the $10^{3}-\mathrm{km}$ scale can be described by the primitive equation system under hydrostatic balance. The basic mathematical structure of this system is relatively well understood (Petcu et al. 2009). This is in stark contrast to the nonhydrostatic anelastic system, a standard formulation adopted for convective-scale modeling. ${ }^{3}$ This system is far more difficult to analyze mathematically, and hence it is much less well known.

The synoptic-scale weather system can, furthermore, be approximated by quasigeostrophy or, alternatively and better, by semigeostrophy, based on the fact that the system exhibits a close balance between the Coriolis and the pressure-gradient forces. This basic feature enables us to understand, to a large extent, synoptic-scale weather in terms of balanced dynamics (cf. Leith 1980).

Unfortunately, under the convective-scale regime, we lose these basic balances of the system, making it much harder to understand the fundamental characteristics of the system. Even a basic proof of nonsingularity associated with latent heating has only recently been established for the simplest case (Temam and Tribbia 2014). Understanding of these flows may partially be accomplished by identifying a wide variety of subsystems defined as asymptotic limits. However, such an understanding requires a much broader knowledge of fluid dynamics and thermodynamics, even without considering full microphysics, than for the traditional synoptic-scale system. However, these subsystems under various asymptotic limits occupy only a small fraction of the vast parameter space in the convective-scale regime. No asymptotic representation is likely to be identified in a bulk part of this regime.

Though all these aspects may sound purely mathematical, our lack of understanding at this most basic level hinders crucial progress at more practical levels (see "Numerics" section).

Dynamical system. Synoptic-scale flows may be understood as a type of dynamical system because mathematically they reside on a slow stable manifold (Leith 1980), which is only weakly coupled to the much more complex dynamics of smaller-scale convection. Thus, dynamics on these scales can be described with a relatively limited number of effective degrees of freedom, that is, low-dimensional dynamics like Lorenz's (1963) strange attractor. Furthermore, such an effective low dimensionality of the system guarantees relatively stable, reliable, long-term model forecasts, even though the evolution may be somehow chaotic.

In the convective-scale regime, on the other hand, although a wide variety of asymptotic regimes emerge, nothing equivalent to geostrophic balance is found: the effective dimension of the system is suddenly increased. As a result, the dynamical system approach mostly developed for low-dimensional systems no longer works effectively. Furthermore, this transition severely restricts predictability (see "Probability" section).

Turbulence. Atmospheric flows are turbulent at almost all the scales of practical interest according to a standard definition of turbulence in fluid mechanics based on the Reynolds number, which measures the importance of nonlinearity relative to viscous dissipation (e.g., Fritsch 1995). Unfortunately, this feature is often neglected due to a custom of calling planetary boundary layer (PBL) turbulence "atmospheric turbulence," leaving an impression that turbulence is only found in the PBL of the atmosphere. It is also typical that a distinction is made between turbulence and convection, which further adds to the impression that atmospheric convection is not turbulent. While the nature of turbulence within convective cells is non-Kolmogorov, and so has different properties to that typically found in the PBL, it is fundamentally a turbulent process.

\footnotetext{
${ }^{3}$ Strictly speaking, many operational models do not follow the anelastic formulation, but adopt the fully compressible formulation. However, these models are still designed not to fully resolve the sound waves by adopting semi-implicit methods for the time integration.
} 
At the synoptic scale, the turbulent nature of the flow is limited by the stratification and rotation of the atmosphere and so tends to be quasi two-dimensional. An important feature of twodimensional turbulence is that the energy is overall transferred from smaller scales to larger scales (an inverse cascade). As a result, atmospheric flows tend to be organized at larger scales, which maintains a relative smoothness of the flow (cf. Tennekes 1978). This property of two-dimensional turbulence allows us to treat synoptic-scale flows as a low-dimensional dynamical system.

On the other hand, once the horizontal scale of the system reaches below $O(10) \mathrm{km}$, the aspect ratio of the flow becomes unity, ${ }^{4}$ hydrostatic balance is no longer satisfied, there is no longer constraint from rotation, and the flow becomes fully threedimensional: this is the essence of the convective scale. These flows are far more complex than two-dimensional turbulence, more transient and intermittent (i.e., they lack balance), and they are associated with a much larger degree of freedom. Thus, three-dimensional turbulent flows are much harder to predict than the chaotic system found in low-dimensional dynamical systems: in the fully turbulent regime, the number of active modes keeps increasing with increasing resolution and prediction becomes increasingly harder with no sign of convergence.

To understand fully three-dimensional convective atmospheric turbulence, the basic nature of the energy interactions between these many active modes in the system should first be properly understood. In fully three-dimensional turbulence, energy is predicted to be transferred overall to the smaller scales, but some of the energy at smaller scales is also transferred to the larger scales, leading to a tendency for organized convection. Although the basic mechanism of organized atmospheric convection is classically attributed to vertical wind shear (cf. Moncrieff and Green 1972; Thorpe et al. 1982; Rotunno et al. 1988), its full mechanism from a point of view of full turbulence dynamics is still to be established (cf. Yano et al. 2012). Here, we also need to move beyond a conventional framework of interactions between convection and the large scale toward a true multiscale framework.
Our current understanding of turbulent flows is essentially based on a straightforward extrapolation of Kolmogorov's theory for homogeneous, threedimensional turbulence (cf. Zilitinkevich et al. 2013). Existence of the stratification and an active role of buoyancy are likely to qualitatively change the basic nature of the flow. Such an investigation into the fundamental nature of self-organized turbulence has not yet been accomplished.

Predictability. The predictability of atmospheric flows is fundamentally limited because the errors in prediction exceed the typical amplitude of a signal of a given scale at a certain point in time. Once the error exceeds this amplitude, the prediction loses any practical value, although it is always possible to run an NWP model beyond this limit.

The fully turbulent nature of the convective-scale regime limits the predictability more severely than for low-dimensional chaotic flows (cf. Palmer et al. 2014). In a chaotic system, an error of the initial condition limits the predictability. In principle, the predictability can always be extended by defining the initial condition more accurately. However, in a fully turbulent regime, the accuracy of the initial condition no longer ultimately limits the predictability (Sun and Zhang 2016), although a denser observational network may extend the predictability to some extent. Rather, the intrinsic nature of the flow itself (notably its intermittency) becomes the ultimate limiting factor. More observations by, for example, a denser network, do not overcome this intrinsic predictability limit.

On the other hand, one may wish that the predictability of synoptic scale would be improved by explicitly resolved convection rather than an unreliable parameterized convection. However, even this is not obvious considering the complex multiple-scale interactions of the turbulent flows associated with convection (see "Turbulence" section).

Probability. The predictability of convective systems is about a few hours (e.g., Hoheneger and Schär 2007), but this is not a fixed number. In some situations, the convective system is strongly controlled by a synoptic-scale process, giving a longer predictability. It is also spatially dependent. Detailed surface data (vegetation, soil types, topography) may further help

\footnotetext{
${ }^{4}$ Observation (cf. Nastrom and Gage 1985) shows that the slope of the kinetic energy spectrum as a function of the wavenumber $k$ turns from $k^{-3}$, as expected for the two-dimensional turbulence, to $k^{-5 / 3}$ at about the few-hundred-kilometer scale (roughly corresponding to the radius of the deformation) in a virtual contradiction to this aspect ratio argument. This regime with a $k^{-5 / 3}$ spectrum above the $10-\mathrm{km}$ scale (often called "stratified turbulence") is still quasi two-dimensional, arising from a strong influence of the stratification on this scale range (cf. Lindborg 2006).
} 
to extend the predictability. Identifying situations with enhanced predictability is an important forecast issue in convective-scale NWP.

However, regardless of its precise value, there always exists a limit beyond which a forecast becomes so uncertain that it loses any deterministic usefulness. As a result, when an NWP model is run for a few days, as is the basic strategy of the NWP community (e.g., ALADIN, COSMO, HIRLAM, Met Office), the resulting forecast can only be interpreted in terms of probabilities: we cannot say precisely when and where an afternoon shower should be expected on the next day, but only give a probability distribution in time and space. In this manner, convective-scale NWP must be inherently based on probability.

Unfortunately, probability is not an easy concept to understand. ${ }^{5}$ It is true that there are already many methodologies for predicting the probability of weather events (e.g., Schwartz et al. 2010). A typically adopted approach is to estimate a probability by creating a large sample or ensemble. However, the frequency of an event within a certain sample is not equivalent to a probability of a single unique event of particular interest. Such frequency-based thinking may be helpful for analyzing a homogeneous sequence of tries (or events), such as the tossing of a coin or dice. In contrast, a sequence of rainfall events is hardly "homogeneous": each event happens under unique circumstances. In this case, a different probability must be assigned for each rainfall event, without creating a sample.

The current standard methodology for estimating weather probabilities, the ensemble prediction system (EPS), is also based on this sample space-based thinking (cf. Leith 1974). Although the EPS is indeed a useful approach, it does not predict by itself a probability in any obvious manner: 3 rain forecasts out of 10 ensemble members does not automatically mean a $30 \%$ chance of rain, unless the sample is defined in a homogeneous manner. Generating such a homogeneous sample with a reasonable, finite ensemble size is not a simple matter, and it becomes more difficult for a system with an increasing number of unstable modes (cf. Uboldi and Trevisan 2015).

Frequency and probability must carefully be distinguished from each other, as Bayesian probability teaches us (cf. Jaynes 2003). Furthermore, any probabilistic prediction system should be derived, ideally, from the basic physical principle for predicting probability, that is, the Liouville equation (Yano and Ouchtar 2017), although its practical use may appear difficult (see "Data assimilation" section).

Stochasticity. Prediction of individual convective events is so difficult that it is tempting to deal with them as random events arising from stochasticities. Such a formulation also more naturally leads to a probabilistic description. However, we have to be cautious in proceeding in this manner.

Some of the physical processes may be intrinsically stochastic: Brownian motion is a classic example. Many complex microphysical processes that do not provide simple closed analytical expressions, for example, generation rate of the secondary ice crystals by a collision of two ice particles (Yano and Phillips 2016), may also be best considered to be stochastic. Following this line of reasoning, one may wish to consider any noisiness in a system as a consequence of stochasticity. However, such reasoning is not necessarily justified. For example, although turbulent flows are extremely noisy, their physics is completely deterministic and presented in a closed form by the Navier-Stokes equations: a relatively simple nonlinearity can easily produce a noisy time series. The choice between using a stochastic or nonlinear representation of a given process must therefore be made carefully.

We should also realize that noisiness at short time scales and small spatial scales does not necessarily lead to a stochastic influence at larger scales: the two levels of the processes must be carefully distinguished from each other. The method of homogenization developed under multiscale asymptotic expansions (Pavliotis and Stuart 2007) provides a rigorous procedure for assessing whether the largescale influences of those noise-like features are actually stochastic.

Generally speaking, we should not assume that all the difficulties in predicting the convectivescale regime arise from randomness: adding more stochasticity is not necessarily a solution. We should also carefully distinguish between the intrinsic

\footnotetext{
${ }^{5}$ Note that the probability is even not a measurable quantity. For example, if a $30 \%$ probability of rain is verified by actual rain $30 \%$ of the time, this probability forecast is statistically consistent with the observation. However, this is not a sufficient condition to verify it. The true verification must be performed on the probability forecast for each event (or nonevent) individually. Of course, this is not possible, because the actual realization is rain or no rain without an intermediate state. In other words, we can never measure a probability observationally for an individual event, but only in a statistical sense. However, the latter is not sufficient for the verification.
} 
stochasticity in physics and the stochasticity introduced as an artificial device in parameterizations. The latter must be addressed with more mathematical rigor (cf. Berner et al. 2017).

Data assimilation. As the horizontal resolution of NWP models increases, a denser observational network is also required. However, simply increasing the number of observations is not enough. NWP models require more information than is being measured: observations generally do not cover the entire model domain, and more importantly, observed quantities are often only indirectly related to model variables. Methodologies for estimating the model state from observations come from nonlinear filtering and optimal control theory (Jazwinski 1970; Crisan and Rozovskii 2011), also referred to as data assimilation (DA; cf. Kalnay 2002) in geosciences.

The full problem of DA consists of estimating the so-called posterior probability: that is, the probability of the model system state based on the observations as well as on our general knowledge of the system (prior information). This problem can be formally solved by invoking the Bayesian theorem (cf. Jaynes 2003). The Liouville equation (or its generalization including stochastic forcing) predicts the time evolution of the probability. However, such a formal approach has so far been seen as unsuitable for NWP applications: the vast dimension of the systems involved renders impractical even just estimating the probabilities, let alone computing their time evolution.

To simplify the problem, Gaussian approximation has often been introduced so that only the mean and covariance of the uncertainty probability must be computed. The two most widely adopted DA methods for operational NWP, four-dimensional variational data assimilation (4DVar: Talagrand and Courtier 1987) and the ensemble Kalman filter (EnKF; Evensen 2009), adopt this simplification. To be even more practical, operational DA is further simplified by tuning the DA to just a single dominant scale, usually the synoptic scale.

On the other hand, as model resolution increases, new phenomena are resolved on a broader range of scales, including convection, and so DA must also be designed to simultaneously keep control on all resolved scales. Studies suggest that this problem may, in principle, be solved by 4DVar (Lorenc and Payne 2007) and EnKF (Snyder and Zhang 2003). However, even more changes in DAs are required to efficiently deal with two main features inherent at the convective scale: i) a much faster and intermittent error growth rate (see "Predictability" section) and ii) the nonlinear and non-Gaussian characters of the underlying dynamics and error statistics.

The first issue is intimately related to the concept of observability (cf. Jazwinski 1970), which may be defined as the problem of identifying the minimum spatiotemporal observational density to efficiently counteract error growth (Quinn and Abarbanel 2010). Observability is a necessary condition for the stability of a DA solution, which is in turn a necessary condition to reduce the state-estimation (and prediction) error (Carrassi et al. 2008). Observability can be achieved through development of the observational network itself as well as of the DA procedure. The former includes, for example, the development of a C-band dual-polarization Doppler radar network under the European Operational Program for Exchange of Weather Radar Information (OPERA; Huuskonen et al. 2014). Surface measurement (e.g., soil moisture) networks with sufficient spatiotemporal resolution also contribute, although they are still to be strengthened over Europe.

There are several approaches for dealing with the second issue, including the rank histogram filter applied to Kalman filter methods (Anderson 2010). However, the most fundamental approach for dealing with this issue is to turn to a more basic principle based on fully Bayesian Monte Carlo methods [particle filters (PFs); Doucet et al. 2000]. A problem with PFs is that the number of particles required for accurate performance grows exponentially as the system's dimension increases (Bocquet et al. 2010). Choosing the importance proposal densities that give a larger overlap with the conditional density may delay the filter collapse, or even prevent it (Slivinski and Snyder 2016). Hybrid EnKF-PF methods are promising alternative approaches to this problem (Chustagulprom et al. 2016). The development of advanced PFs for DA in convection-permitting NWP models will be an important priority for the coming years (cf. Poterjoy et al. 2017).

Cloud microphysics. Increasing model resolution also demands more sophisticated physics. Unfortunately, the issues of physics are vast. Here, we deliberately limit our discussions to the cloud microphysics because of its unique status.

Our knowledge of microphysical processes coming both from laboratory and theoretical studies is quite extensive (cf. Pruppacher and Klett 1997), although our knowledge is hardly perfect and the existing bin microphysics parameterizations certainly do not make full use of this knowledge. At the same time, 
even the current bin microphysical schemes are still too expensive to use for convective-scale NWPs. In short, we know the microphysics too well, and we have to somehow simplify it for it to be included in operational NWP models while maintaining a reasonable model run speed. The main problem with current microphysical modeling is that these simplifications are made in a rather arbitrary manner without performing any systematic "investment gain" analysis. For example, one can find many articles in the literature claiming an improvement of a model by upgrading, for example, from a single-moment to a double-moment scheme. However, a carefully balanced judgment is often missing on relative gain against a given investment. Here, Bayesian decision theory (Berger 1985) may be called for. A solid first step toward this direction is taken by, for example, van Lier-Walqui et al. (2014).

The benefits of implementing more realistic, and more complex, descriptions of cloud microphysics may appear enormous: hail damage could be better estimated by fully considering the hail size and hardness (Phillips et al. 2014), and winter precipitation (due to ice, liquid, or a mixture of both) may be better predicted by using a more detailed description of the melting process (e.g., Phillips et al. 2007). However, in the convective-scale regime, the expected improvements may not be attainable: with convective-scale turbulence intrinsically interacting with the enhanced cloud microphysics, an increase in the complexity of the microphysics may not automatically lead to a more reliable forecast, but may lead merely to higher forecast uncertainties as if adding white noise. A suitable level of sophistication in deterministic physics (not only microphysics, but surface processes, radiation, etc.) must be objectively and quantitatively assessed, with this aspect being fully taken into account.

Parameterization. The role of subgrid-scale parameterizations becomes more subtle as convection starts to become explicitly resolved. In traditional NWP models, individual convective storms are key elements to be parameterized. Under the convection-permitting regime, these parameterizations become almost unnecessary. In fact, most operational convectionpermitting NWP models turn off the deep-convection parameterization. However, the threshold resolution for turning it off is not well established.

It is more likely that the transition toward a situation where it is no longer necessary to parameterize deep convection should be more gradual, and certain intermediate procedures are required in this transition regime (e.g., Gerard et al. 2009). These procedures should be performed without traditional parameterization assumptions such as scale separation and quasi equilibrium. Some studies propose a stochastic formulation (e.g., Plant and Craig 2008), although a formal formulation analysis shows that the system remains deterministic even without these traditional assumptions (Yano 2014).

The focus is likely to shift to the PBL (Ching et al. 2014). However, many new parameterization issues also arise there, including those for subcloud scales of deep convection: it is very likely that the turbulent mixing between the clouds and the immediate environment must be described more carefully than traditional entrainment-detrainment descriptions (cf. de Rooy et al. 2013).

Overall, we face challenges for subgrid-scale parameterizations from two sides. On the one side, we need to further elaborate existing parameterizations (e.g., deep and shallow convection, PBL). On the other side, we also need to introduce new parameterizations, for example, for the subcloudscale processes. It naturally follows that the consistencies between the existing and the new parameterizations must also be carefully established. The interactions between various subgrid-scale processes, for example, between the PBL and convection, also become more critically important.

To effectively tackle all these problems together, we face issues of consistency and unification. Here, we propose that the best solution would be to develop a single consistent unit of subgrid-scale parameterizations by returning to the first principles of explicit physics (e.g., a large-eddy simulation PDE system), and to reconstruct everything from there. For specific procedures, see Yano et al. (2015) and Yano (2016). Rebuilding everything from scratch is often much faster, in the end, than trying to unify something already in place, but developed without much regard for mutual consistencies. These more robust parameterizations are, furthermore, expected to make the subgrid-scale information more practically useful in forecasts (cf. Kain et al. 2010; de Meutter et al. 2015).

Numerics. In the traditional synoptic-scale regime, which in essence resides on a low-dimensional dynamical system, increases in spatial resolution have, overall, contributed to a better convergence of the forecast quality. On the other hand, in the convective-scale regime, with so many modes actively involved in the dynamics, solutions of the governing equations are computable with much 
smaller accuracy at any practical resolution, and the solutions do not converge with increasing resolution. For example, the Met Office Unified Model finds no tendency toward forecast convergence when increasing horizontal grid spacing from $1.5 \mathrm{~km}$ to $100 \mathrm{~m}$ (Stein et al. 2015), since the increase of horizontal resolution gradually resolves more turbulent processes. As a conventional wisdom, grid spacings at least as fine as $O\left(10^{1}-10^{2}\right) \mathrm{m}$ are required for large-eddy simulations (LESs) to be meaningful. The typical convection-permitting grid spacing is only just comparable to the size of the largest eddies within the PBL.

Prominent flow features are often realized right at the limit of the model resolution in convectionpermitting scale simulations, making the simulations sensitive to details of subgrid-scale parameterizations as well as to the properties of the numerical algorithms. As a result, some artifacts in outputs may result. For example, investigating the flow over a heated plane, Piotrowski et al. (2009) find that anisotropic viscosity can artificially produce realisticlooking regular structures that mimic naturally generated Rayleigh-Bernard cells. Clearly, verification of these numerical results critically depends on the availability of theoretically and mathematically correct solutions of the PDEs, which can help provide a more rigorously defined testing and selection of the numerical algorithms suitable for convectionresolving computations.

Among the numerical algorithms, advection is common to every physical variable and therefore of particular importance. A good advection scheme must conserve the sign and the shape of a variable to be advected, when the system is purely advective, by suppressing artificial oscillations and numerical diffusion. Some advection schemes suppress numerical diffusion by introducing an antidiffusion term (limiter). For example, the "flux corrected transport" method, as adopted by, for example, Smolarkiewicz (2006), constructs advective fluxes as weighted averages of a flux computed by a monotonic, but diffusive, low-order scheme and a flux computed by a high-order scheme so as to suppress unphysical behavior.

Semi-Lagrangian schemes (Staniforth and Côté 1991) are popular among NWP models because they permit a relatively large time step while still allowing the model to run smoothly. However, we must be cautious with their application to the turbulent convective-scale regime (cf. Lauritzen et al. 2011). Although some successful turbulent applications may be found in the literature, semi-
Lagrangian schemes work most efficiently for a relatively laminar flow.

In convective-scale turbulent calculations, the numerics must be robust. ${ }^{6}$ Particular attention is required for the dynamical core, including the treatment of advection. Though no explicit discussion is provided herein, attention must also be equally paid to the numerical solver for the physics and the subgrid-scale parameterization (Dubal et al. 2006; Termonia and Hamdi 2007).

CONCLUSIONS. We have identified the following fundamental theoretical challenges in convectivescale NWPs:

- PDE: A lack of proper understanding both of the dynamics and the partial differential equations describing this regime poses serious difficulty, especially for the verification of numerical model results.

- Turbulence: A theory of turbulence must be developed going beyond the traditional approaches based on relatively straightforward extrapolation of Kolmogorov's theory for homogeneous turbulence, to the buoyancy-driven stratified case.

- Probability: Probability becomes a key variable to be predicted, because NWP models are run for much longer time scales (a few days) than the predictability limit (a few hours). The intrinsic probability, as defined by the Bayesian probability theory, should be evaluated rather than the oft-used estimation of probability by frequency counting. The Liouville equation, as a basic physical principle of probability prediction, should be further exploited to accomplish this.

- Data assimilation: New assimilation approaches such as the particle filters must be pursued because the traditional assumptions of quasi linearity and Gaussian distributions are no longer valid.

- Observational network: Although the development of a denser observational network may be crucial, it is meaningful only under the constraints of observability. Moreover, the intrinsic limit of predictability (a few hours) due to the fully turbulent nature of the convective-scale regime ultimately prevents us from extending

\footnotetext{
${ }^{6}$ In certain situations, "robust" only narrowly refers to whether a given scheme is conditionally stable. On the other hand, here we use this notion in the more general sense that given numerics are not only stable, and insensitive to a change of the resolution, etc., but also preserve the basic numerical properties predicted by theory.
} 
predictability through the inclusion of more observations.

- Stochasticity: Stochasticity must be introduced into forecast models in a more robust and solid manner, for example, based on the method of homogenization under multiscale asymptotic expansions. It is important to keep in mind that more than a mere existence of fluctuations is required to justify the introduction of stochasticity into physics.

- Physics: The degree of sophistication of the model physics, notably of the cloud microphysics, must be decided by investment-gain analysis, for example, based on Bayesian decision theory. Some of the physical processes may be better represented simply as a stochasticity.

- Parameterizations: Subgrid-scale parameterizations should be redeveloped from scratch in a unified manner, starting from a basic set of equations for the physics and dynamics, as given by, for example, LES models, so that universality and consistency are ensured.

- Numerics: The fully turbulent nature of the convective-scale regime demands that the numerical algorithms be much more robust than in traditional NWP models, especially to avoid generation of artificially organized structures at the scale of the model resolution.

Each research direction requires its own substantial investments, augmenting current efforts and being subject to development of more detailed research strategies. We do not even pretend that these investigations are easy. For example, at this stage, it would be impossible to make any progress with the convective-scale regime as a PDE problem if the traditional, rigorous methodologies are to be applied; a completely different approach would be required here. On the other hand, the assimilation problem can be addressed more easily as a continuation of the current efforts. Intensive investments into the currently existing top-end methodologies are likely to lead to breakthroughs in the relatively short term.

It is also crucial to extensively exploit existing knowledge from non-atmospheric science literature, for example, from turbulence research. These fundamental scientific issues require our rethinking and restructuring, but also redirecting of some non-atmospheric science research to more fundamental problems. For example, non-Kolmogorov turbulence is not solely an atmospheric problem, but it has much wider applications. A well-organized research network, as well as supporting funding, would be required so that highly multidisciplinary research may be formed to address these problems in full.

ACKNOWLEDGMENTS. J.I.Y. appreciates extensive discussions with European consortia members as well as a number of mathematicians over the years, which led to this essay. The authors gratefully acknowledge all reviewers, including Andrew Lorenc and Lou Wicker, for their insightful comments and suggestions, which improved the clarity and presentation of this essay. The opinions expressed in the present essay do not necessarily reflect the official positions of the consortia, the agencies, or the institutions that the authors are affiliated with. A.C. has been funded by the project REDDA of the Norwegian Research Council.

\section{REFERENCES}

Anderson, J. L., 2010: A non-Gaussian ensemble filter update for data assimilation. Mon. Wea. Rev., 138, 4186-4198, https://doi.org/10.1175/2010MWR3253.1.

Bauer, P., A. Thorpe, and G. Brunet, 2015: The quiet revolution of numerical weather prediction. Nature, 525, 47-55, https://doi.org/10.1038/nature14956.

Berger, J. O., 1985: Statistical Decision Theory and Bayesian Analysis. 2nd ed. Springer, 617 pp.

Berner, J., and Coauthors, 2017: Stochastic parameterization: Towards a new view of weather and climate models. Bull. Amer. Meteor. Soc., 98, 565-588, https:// doi.org/10.1175/BAMS-D-15-00268.1.

Bocquet, M., C. A. Pires, and L. Wu, 2010: Beyond Gaussian statistical modeling in geophysical data assimilation. Mon. Wea. Rev., 138, 2997-3023, https://doi.org /10.1175/2010MWR3164.1.

Carrassi, A., M. Ghil, A. Trevisan, and F. Uboldi, 2008: Data assimilation as a nonlinear dynamical systems problem: Stability and convergence of the predictionassimilation system. Chaos, 18, 023112, https://doi .org/10.1063/1.2909862.

Ching, J., R. Rotunno, M. LeMone, A. Martilli, B. Kosovic, P. A. Jimenez, and J. Dudhia, 2014: Convectively induced secondary circulations in fine-grid mesoscale numerical weather prediction models. Mon. Wea. Rev., 142, 3284-3302, https://doi .org/10.1175/MWR-D-13-00318.1.

Chustagulprom, N., S. Reich, and M. Reinhardt, 2016: A hybrid ensemble transform particle filter for nonlinear and spatially extended dynamical systems. SIAM/ ASA J. Uncertainty Quantif., 4, 592-608, https://doi .org/10.1137/15M1040967.

Crisan, D., and B. Rozovskii, 2011: The Oxford Handbook of Nonlinear Filtering. Oxford University Press, $1080 \mathrm{pp}$. 
de Meutter, P., L. Gerard, G. Smet, K. Hamid, R. Hamdi, D. Degrauwe, and P. Termonia, 2015: Predicting small-scale, short-lived downbursts: Case study with the NWP limited-area ALARO model for the Pukkelpop thunderstorm. Mon. Wea. Rev., 143, 742756, https://doi.org/10.1175/MWR-D-14-00290.1.

de Rooy, W. C., and Coauthors, 2013: Entrainment and detrainment in cumulus convection: An overview. Quart. J. Roy. Meteor. Soc., 139, 1-19, https://doi .org/10.1002/qj.1959.

Doucet, A., S. Godskill, and C. Anrieu, 2000: On sequential Monte Carlo sampling methods for Bayesian filtering. Stat. Comput., 10, 197-208, https://doi.org/10.1023 /A:1008935410038.

Dubal, M., N. Wood, and A. Staniforth, 2006: Some numerical properties of approaches to physicsdynamics coupling for NWP. Quart. J. Roy. Meteor. Soc., 132, 27-42, https://doi.org/10.1256/qj.05.49.

Evensen, G., 2009: Data Assimilation: The Ensemble Kalman Filter. 2nd ed. Springer, 306 pp.

Fritsch, J. M., and R. E. Carbone, 2004: Improving quantitative precipitation forecasts in the warm season: A USWRP research and development strategy. Bull. Amer. Meteor. Soc., 85, 955-965, https://doi.org/10.1175 /BAMS-85-7-955.

Fritsch, U., 1995: Turbulence: The Legacy of A. N. Kolmogorov. Cambridge University Press, 296 pp.

Gerard, L., J.-M. Piriou, R. Brožková, J.-F. Geleyn, and D. Banciu, 2009: Cloud and precipitation parameterization in a meso-gamma-scale operational weather prediction model. Mon. Wea. Rev., 137, 3960-3977, https://doi.org/10.1175/2009MWR2750.1.

Hoheneger, C., and C. Schär, 2007: Atmospheric predictability at synoptic versus cloud-resolving scales. Bull. Amer. Meteor. Soc., 88, 1783-1793, https://doi .org/10.1175/BAMS-88-11-1783.

Huuskonen, A., E. Saltikoff, and I. Holleman, 2014: The operational weather radar network in Europe. Bull. Amer. Meteor. Soc., 95, 897-907, https://doi .org/10.1175/BAMS-D-12-00216.1.

Jaynes, E. T., 2003: Probability Theory: The Logic of Science. Cambridge University Press, 727 pp.

Jazwinski, A. H., 1970: Stochastic Processes and Filtering Theory. Academic Press, 376 pp.

Kain, J. S., S. R. Dembek, S. J. Weiss, J. L. Case, J. J. Levit, and R. A. Sobash, 2010: Extracting unique information from high resolution forecast models: Monitoring selected fields and phenomena every time step. Wea. Forecasting, 25, 1536-1542, https:// doi.org/10.1175/2010WAF2222430.1.

Kalnay, E., 2002: Atmospheric Modeling, Data Assimilation and Predictability. Cambridge University Press, $368 \mathrm{pp}$.
Lauritzen, P. H., P. A. Ullrich, and R. D. Nair, 2011: Atmospheric transport schemes: Desirable properties and a semi-Lagrangian view on finite-volume discretizations. Numerical Techniques for Global Atmospheric Models, P. H. Lauritzen et al., Eds., Lecture Notes in Computational Science and Engineering, Vol. 80, Springer, 185-250.

Leith, C. E., 1974: Theoretical skill of Monte Carlo forecasts. Mon. Wea. Rev., 102, 409-418, https:// doi.org/10.1175/1520-0493(1974)102<0409:TSOMCF $>2.0 . \mathrm{CO} ; 2$.

- 1980: Nonlinear normal mode initialization and quasi-geostrophic theory. J. Atmos. Sci., 37, 958-968, https://doi.org/10.1175/1520-0469(1980) 037<0958:NNMIAQ>2.0.CO;2.

Lindborg, E., 2006: The energy cascade in a strongly stratified fluid. J. Fluid Mech., 550, 207-242, https:// doi.org/10.1017/S0022112005008128.

Lorenc, A. C., and T. Payne, 2007: 4D-Var and the butterfly effect: Statistical four-dimensional data assimilation for a wide range of scales. Quart. J. Roy. Meteor. Soc., 133, 607-614, https://doi.org/10.1002/qj.36.

Lorenz, E. N., 1963: Deterministic nonperiodic flow. J. Atmos. Sci., 20, 130-141, https://doi.org/10.1175/1520 $-0469(1963) 020<0130: \mathrm{DNF}>2.0 . \mathrm{CO} ; 2$.

Mass, C. F., 2006: The uncoordinated giant. Bull. Amer. Meteor. Soc., 87, 573-584, https://doi.org/10.1175 /BAMS-87-5-573.

- D. Ovens, K. Westrick, and B. A. Colle, 2002: Does increasing horizontal resolution produce more skillful forecasts? Bull. Amer. Meteor. Soc., 83, 407-430, https://doi.org/10.1175/1520-0477(2002)083<0407: DIHRPM $>2.3 . \mathrm{CO} ; 2$.

Moncrieff, M. W., and J. S. A. Green, 1972: The propagation and transfer properties of steady convective overturning in shear. Quart. J. Roy. Meteor. Soc., 98, 336-352, https://doi.org/10.1002/qj.49709841607.

Nastrom, G. D., and K. S. Gage, 1985: A climatology of atmospheric wavenumber spectra of wind and temperature observed by commercial aircraft. $J$. Atmos. Sci., 42, 950-960, https://doi.org/10.1175/1520 -0469(1985)042<0950:ACOAWS>2.0.CO;2.

Palmer, T. N., A. Döring, and G. Seregin, 2014: The real butterfly effect. Nonlinearity, 27, R123-R141, https:// doi.org/10.1088/0951-7715/27/9/R123.

Pavliotis, G. A., and A. M. Stuart, 2007: Multiscale Methods: Averaging and Homogenization. Springer, $307 \mathrm{pp}$.

Petcu, M. R., M Temam, and M. Ziane, 2009: Some mathematical problems in geophysical fluid dynamics. Special Volume: Computational Methods for the Atmosphere and the Oceans, R. M. Temam and J. J. Tribbia, Eds., Vol. 14, Handbook of Numerical 
Analysis, Elsevier, 577-750, https://doi.org/10.1016 /S1570-8659(08)00212-3.

Phillips, V. T. J., A. Pokrovsky, and A. Khain, 2007: The influence of time-dependent melting on the dynamics and precipitation production in maritime and continental storm-clouds. J. Atmos. Sci., 64, 338-359, https://doi.org/10.1175/JAS3832.1.

—, A. Khain, N. Benmoshe, and E. Ilotovich, 2014: Theory of time-dependent freezing and its application in a cloud model with spectral bin microphysics. I: Wet growth of hail. J. Atmos. Sci., 71, 4527-4557, https://doi.org/10.1175/JAS-D-13-0375.1.

Piotrowski, Z. P., P. K. Smolarkiewicz, S. P. Malinowski, and A. A. Wyszogrodzki, 2009: On numerical realizability of thermal convection. J. Comput. Phys., 228, 6268-6290, https://doi.org/10.1016/j .jcp.2009.05.023.

Plant, R. S., and G. C. Craig, 2008: A stochastic parameterization for deep convection based on equilibrium statistics. J. Atmos. Sci., 65, 87-105, https://doi .org/10.1175/2007JAS2263.1.

Poterjoy, J., R. A. Sobash, and J. L. Anderson, 2017: Convective-scale data assimilation for the weather research and forecasting model using the local particle filter. Mon. Wea. Rev., 145, 1897-1918, https:// doi.org/10.1175/MWR-D-16-0298.1.

Pruppacher, H. R., and J. D. Klett, 1997: Microphysics of Clouds and Precipitation. Kluwer Academic Publishers, $712 \mathrm{pp}$.

Quinn, J. C., and H. D. Abarbanel, 2010: State and parameter estimation using Monte Carlo evaluation of path integrals. Quart. J. Roy. Meteor. Soc., 136, 1855-1867, https://doi.org/10.1002/qj.690.

Rotunno, R., J. B. Klemp, and M. L. Weisman, 1988: A theory for strong, long-lived squall lines. J. Atmos. Sci., 45, 463-485, https://doi.org/10.1175/1520-0469(1988) 045<0463:ATFSLL >2.0.CO;2.

Schwartz, C. S., and Coauthors, 2010: Toward improved convection-allowing ensembles: Model physics sensitivities and optimizing probabilistic guidance with small ensemble membership. Wea. Forecasting, 25, 263-280, https://doi.org /10.1175/2009WAF2222267.1.

Slivinski, L., and C. Snyder, 2016: Exploring practical estimates of the ensemble size necessary for particle filters. Mon. Wea. Rev., 144, 861-875, https://doi .org/10.1175/MWR-D-14-00303.1.

Smolarkiewicz, P., 2006: Multidimensional positive definite advection transport algorithm: An overview. Int. J. Numer. Methods Fluids, 50, 1123-1144, https:// doi.org/10.1002/fld.1071.

Snyder, C., and F. Zhang, 2003: Assimilation of simulated Doppler radar observations with an ensemble
Kalman filter. Mon. Wea. Rev., 131, 1663-1677, https://doi.org/10.1175//2555.1.

Staniforth, A., and J. Côté, 1991: Semi-Lagrangian integration schemes for atmospheric models-A review. Mon. Wea. Rev., 119, 2206-2223, https:// doi.org/10.1175/1520-0493(1991)119<2206:SLISFA $>2.0 . \mathrm{CO} ; 2$.

Stein, T. H. M., and Coauthors, 2015: The DYMECS Project: A statistical approach for the evaluation of convective storms in high-resolution NWP models. Bull. Amer. Meteor. Soc., 96, 939-951, https://doi .org/10.1175/BAMS-D-13-00279.1.

Stensrud, D. J., and Coauthors, 2009: Convectivescale warn-on-forecast system: A vision for 2020 . Bull. Amer. Meteor. Soc., 90, 1487-1499, https://doi .org/10.1175/2009BAMS2795.1.

Sun, J., and Coauthors, 2014: Use of NWP for nowcasting convective precipitation. Bull. Amer. Meteor. Soc., 95, 409-426, https://doi.org/10.1175/BAMS -D-11-00263.1.

Sun, Y., and F. Zhang, 2016: Intrinsic versus practical limits of atmospheric predictability and the significance of the butterfly effect. J. Atmos. Sci., 73, 1419-1438, https://doi.org/10.1175/JAS-D-15 -0142.1 .

Talagrand, O., and P. Courtier, 1987: Variational assimilation of meteorological observations with the adjoint vorticity equation. I: Theory. Quart. J. Roy. Meteor. Soc., 113, 1311-1328, https://doi.org/10.1002 /qj.49711347812.

Temam, R., and J. Tribbia, 2014: Uniqueness of solutions for moist advection problems. Quart. J. Roy. Meteor. Soc., 140, 1315-1318, https://doi.org/10.1002 /qj.2217.

Tennekes, H., 1978: Turbulent flow in two and three dimensions. Bull. Amer. Meteor. Soc., 59, 22-28, https://doi.org/10.1175/1520-0477(1978)059<0022: TFITAT>2.0.CO;2.

Termonia, P., and R. Hamdi, 2007: Stability and accuracy of the physics-Dynamics coupling in spectral models. Quart. J. Roy. Meteor. Soc., 133, 1589-1604, https://doi.org/10.1002/qj.119.

Thorpe, A. J., M. J. Miller, and M. W. Moncrieff, 1982: Two-dimensional convection in non-constant shear: A model of mid-latitude squall lines. Quart. J. Roy. Meteor. Soc., 108, 739-762, https://doi.org/10.1002 /qj.49710845802.

Uboldi, F., and A. Trevisan, 2015: Multiple-scale error growth in a convection-resolving model. Nonlinear Processes Geophys., 22, 1-13, https://doi.org/10.5194 /npg-22-1-2015.

van Lier-Walqui, M., T. Vukicevic, and D. J. Posselt, 2014: Linearization of micropysical parameterization 
uncertainty using multiplicative process perturbation parameters. Mon. Wea. Rev., 142, 401-413, https://doi.org/10.1175/MWR-D-13-00076.1.

Yano, J.-I., 2014: Formulation structure of the massflux convection parameterization. Dyn. Atmos. Oceans, 67, 1-28, https://doi.org/10.1016/j.dynatmoce 2014.04.002.

_, 2016 : Subgrid-scale physical parameterization in atmospheric modelling: How can we make it consistent? J. Phys. A: Math. Theor., 49, 284001, https://doi .org/10.1088/1751-8113/49/28/284001.

— low and deep convection under a finite departure from convective quasi-equilibrium. J. Atmos. Sci., 69, 3463-3470, https://doi.org/10.1175/JAS-D-12 -0108.1 .

— , and V. T. J. Phillips, 2016: Explosive ice multiplication induced by multiplicative-noise fluctuation of mechanical breakup in ice-ice collisions. J. Atmos. Sci., 73, 4685-4697, https://doi.org/10.1175/JAS -D-16-0051.1.
- and E. Ouchtar, 2017: Convective initiation uncertainties without trigger or stochasticity: Probabilistic description by the Liouville equation and Bayes' theorem. Quart. J. Roy. Meteor. Soc., 143, 2025-2035, https://doi.org/10.1002/qj.3064.

—, C. Liu, and M. W. Moncrieff, 2012: Self-organized criticality and homeostasis in atmospheric convective organization. J. Atmos. Sci., 69, 3449-3462, https:// doi.org/10.1175/JAS-D-12-069.1.

—, L. Bengtsson, J.-F. Geleyn, and R. Brozkova, 2015: Towards a unified and self-consistent parameterization framework. Parameterization of Atmospheric Convection, Vol. 2, R. S. Plant and J.-I. Yano, Eds., World Scientific, 423-435, https://doi .org/10.1142/9781783266913_0030.

Zilitinkevich, S. S., T. Elperin, N. Kleeorin, I. Rogachevskii, and I. Esau, 2013: A hierarchy of energy- and flux-budget (EFB) turbulence closure models for stably-stratified geophysical flows. Bound.-Layer Meteor., 146, 341-373, https://doi .org/10.1007/s10546-012-9768-8.

\section{CLIMATE CHANGE/POLICY}

“ This book is timely because global climate change policy is a mess.... Drawing on concrete examples and a broad range of social science theory, this book convincingly makes the case for a social learning approach to both adaptation and emissions mitigation."

- Steve Rayner, James Martin Professor of Science and Civilization, University of Oxford

\section{Adaptive Governance and Climate Change \\ RONALD D. BRUNNER AND AMANDA H. LYNCH}

As greenhouse gas emissions and temperatures at the poles continue to rise, so do damages from extreme weather events affecting countless lives. Meanwhile, ambitious international efforts to cut emissions have proved to be politically ineffective or infeasible. There is hope, however, in adaptive governance-an approach that has succeeded in some communities and can be undertaken by others around the globe.

In this book:

- A political and historical analysis of climate change policy

- How adaptive governance works on the ground

- Why local, bottom-up approaches should complement global-scale negotiations

(C) 2010, PAPERBACK, 424 PAGES

ISBN: 978-1-878220-97-4

AMS CODE: AGCC

LIST \$35 MEMBER \$22 